\title{
Pharmacological suppression of the Ras/MAPK pathway in thyroid carcinoma cells can provoke opposite effects on cell migration and proliferation: The appearance of yin-yang effects and the need of combinatorial treatments
}

\author{
ALEXANDER GLASSMANN $^{1^{*}}$, JOCHEN WINTER $^{2^{*}}$, DOMINIK KRAUS $^{3}$, \\ NADINE VEIT ${ }^{4}$ and RAINER PROBSTMEIER ${ }^{4}$ \\ ${ }^{1}$ Life Science Inkubator GmbH; ${ }^{2}$ Oral Cell Biology Group, Department of Periodontology, Operative and Preventive Dentistry, \\ ${ }^{3}$ Department of Prosthodontics, Preclinical Education and Material Science, University of Bonn; ${ }^{4}$ Neuro- and \\ Tumor Cell Biology Group, Department of Nuclear Medicine, University Hospital of Bonn, Bonn, Germany
}

Received June 13, 2014; Accepted August 1, 2014

DOI: $10.3892 /$ ijo.2014.2668

\begin{abstract}
A major challenge in tumor therapy is the decrease or even the halting of cell proliferation and migration of cancerous cells. In the present study, we have analyzed the impact of a pharmacological blockade of the PI3K/Akt and MAPK/ERK1/2 signaling pathways on cell migration, proliferation and cell death in three human thyroid tumor cell lines that represent the main types of malignant thyroid carcinomas (B-CPAP, follicular; Cal-62, anaplastic; FTC-133, papillary thyroid carcinoma cells) and in which these pathways are constitutively activated. In general, pharmacological perturbation of PI3/Akt (application of MK-2206) and MEK/ ERK1/2 (application of PD0325901 or U0126) signaling led to a cell line and drug-specific decrease in the proliferation and migration potential of thyroid carcinoma cells, although to a varying extent. However, one exception became apparent: in Cal-62 cells inhibition of the MEK/ERK1/2 module increased the migration rate up to $50 \%$. This effect could be prevented by a simultaneous suppression of the PI3/Akt pathway, but also by application of the multiple kinase inhibitor sorafenib, a treatment that did not change the activation state of Akt. Thus, a pharmacological perturbation of canonical signaling pathways in thyroid carcinoma may induce drug-dependent yin-yang effects that are characterized by a simultaneous suppression of one (i.e., proliferation) and the activation of another (i.e., migration) cellular process. The appearance of
\end{abstract}

Correspondence to: Dr Rainer Probstmeier, Neuro- and Tumor Cell Biology Group, Department of Nuclear Medicine, University Hospital of Bonn, Sigmund-Freud-Str. 25, D-53127 Bonn, Germany

E-mail: r.probstmeier@uni-bonn.de

*Contributed equally

Key words: Akt, ERK1/2, thyroid cancer, migration, proliferation, small molecule kinase inhibitor such phenomena should be taken into account when therapy plans are established.

\section{Introduction}

Well-differentiated papillary and follicular carcinomas typically have indolent behavior and can be effectively treated by surgery followed by radioiodine therapy (1). However, tumors that lose differentiation during progressive disease or already have developed as undifferentiated entities (i.e., anaplastic thyroid tumors) have lost the ability to trap iodine. As a consequence, these types of tumors do not respond to conventional radioiodine treatment and, thus, have a much less favorable prognosis (2). Hence, alternative concepts are urgently warranted to improve therapeutic undertakings. In recent years, small molecule kinase inhibitors have been introduced in therapeutic treatments of several types of cancers $(3,4)$. Especially in regard to thyroid cancers focus has been directed to the Ras/ERK1/2 and PI3K/AKT pathways because both are activated in a high percentage of thyroid tumors. In more than $80 \%$ of papillary cancers, for example, genes of the MAPK pathway are affected (5). Activation of the classical MAPK pathway occurs mainly via genetic alterations of Ras, Raf or Ret genes (6). In addition to the activation of the MAPK pathway, RET/PTC or BRAF mutations can also result in the activation of $\mathrm{PI} 3 \mathrm{~K}$. In thyroid cancer, moreover, the activation of Akt can also be a result of PI3K mutation and amplification, RET/PTC rearrangements, reduction or loss of PTEN expression (7).

Clinical studies with specific ERK1/2 or Akt inhibitors as well as with multikinase inhibitors such as sorafenib have already been undertaken with promising results. Sorafenib (BAY 43-9006, Nexavar) is a second-generation, orally active multikinase inhibitor that was recently approved for the treatment of patients with advanced renal cell carcinoma and patients with unresectable hepatocellular carcinoma (8). Some clinical studies have also demonstrated the effectiveness of sorafenib in advanced thyroid cancer $(9,10)$. The action of 
sorafenib affects a broader range of signal transducers, such as vascular endothelial growth factor receptors, platelet-derived growth factor receptor- $\beta$, c-kit or RET (8). Sorafenib downregulates activated Akt and ERK1/2 in neuroblastoma (11) and lymphoma cells (12), but, unexpectedly, can also initiate ERK1/2 $(13,14)$ or Akt activation (15) that leads to increased tumor cell proliferation and migration. The use of the MEK inhibitors PD0325901 or U0126 is restricted to academic research, as these drugs produced undesirable side effects in clinical trials (16). Nevertheless, these compounds serve as valuable experimental paradigms to analyze the consequences of MEK/ERK1/2 inactivation on the cellular level.

In the present study we show that in thyroid carcinoma cells downregulation of Ras/MAPK and PI3K/Akt pathway activation predominantly suppresses cell migration and proliferation. In the anaplastic cell line Cal-62, however, inactivation of Ras/MAPK signaling positively affects the migration rate. This increase can be suppressed by PI3K/Akt pathway-dependent (MK-2006 treatment) and, most likely, -independent (sorafenib treatment) mechanisms.

\section{Materials and methods}

Cell lines and culture conditions. Human papillary thyroid cancer cell line B-CPAP and human follicular thyroid cancer cell line FTC-133 were from Professor G. Brabant (Department of Endocrinology, Christie Hospital, Manchester, UK). Human anaplastic thyroid cancer cell line Cal-62 was obtained from Leibniz Institute DSMZ, German Collection of Microorganisms and Cell Cultures (Braunschweig, Germany). B-CPAP cells were cultivated in RPMI-1640 medium, Cal-62 cells in DMEM, and FTC-133 cells in DMEM/Ham's F12. Media were supplemented with $10 \%$ fetal calf serum (FCS), $1 \%$ penicillin-streptomycin and L-glutamine.

Cell extraction and western blot analysis. Cell solubilisation in the presence of protease and phosphatase inhibitors and western blot analyses were carried out as described (17).

Drug treatment. Stock solutions and working concentrations were as follows: for MK-2206 (Selleck Chemicals), $10 \mathrm{mM}$ and $1 \mu \mathrm{M}$, for PD0325901 (Axon Medchem) $10 \mathrm{mM}$ and $2 \mu \mathrm{M}$, for sorafenib (Enzo Life Sciences) $10 \mathrm{mM}$ and $5 \mu \mathrm{M}$, for U0126 (Promega) $10 \mathrm{mM}$ and $20 \mu \mathrm{M}$, for wortmannin (Santa Cruz) $1 \mathrm{mM}$ and $100 \mathrm{nM}$. All stock solutions were prepared in DMSO and stored at $-20^{\circ} \mathrm{C}$. In a typical experiment, cells were cultivated for three days in the absence or presence of the compound that was added once after $36 \mathrm{~h}$ or twice after 24 and $48 \mathrm{~h}$.

Cell proliferation assay. To analyze cell proliferation, 500 cells in $100 \mu$ l culture medium were seeded in 96-well plates (12 wells per time-point and treatment). Drugs were added immediately after 24 as well as after $48 \mathrm{~h}$ in $100 \mu \mathrm{l}$ of culture medium. Addition of pure DMSO served as a medium control. Cells were fixed in $4 \%$ formaldehyde in PBS just before treatment (T0) or after $72 \mathrm{~h}$ of treatment (T1). Cells from both time-points were then stained with DAPI $(1 \mu \mathrm{g} / \mathrm{ml}$ in PBS) in order to determine microscopically the cell number in a defined area of each well.
Collective cell migration assay. Cell suspensions $(5,000$ cells $/ \mu \mathrm{l})$ in a total volume of $3 \mu \mathrm{l}$ were seeded on defined regions in Petri dishes and allowed to adhere for two to three hours. After floating the dishes with culture medium, the adherent and confluent cells occupied a circular area. Diameters of areas (12 per dish) were determined microscopically subsequently after floating (T0) and after a 72-h culture period (T1). Compounds were added at T0 as well as 24 and $48 \mathrm{~h}$ later.

Cell toxicity assay. Cytotoxicity was analyzed with the LDH Cytotoxicity Assay Kit from Roche. Briefly, 10,000 cells (in $100 \mu \mathrm{l}$ culture medium) were seeded per well in 96-well plates. Twenty-four hours later, cells were exposed to different drug concentrations for 24-72 h. After treatment, LDH activity was determined in the cell culture supernatants. In parallel, cells that had been treated identically were lysed in order to determine total LDH activity, a value that allows to quantify cell proliferation.

\section{Results}

ERK1/2 and Akt activation in human thyroid cancer cells. Human papillary cell line B-CPAP, anaplastic cell line CAL-62 and follicular cell line FTC-133 were maintained for $48 \mathrm{~h}$ in the presence or absence of serum, or for $48 \mathrm{~h}$ in the absence and finally for $20 \mathrm{~min}$ in the presence of serum. Under serum-free and serum-containing culture conditions, no considerable differences in the expression levels of pERK1/2 or pAkt were observed (Fig. 1). Stimulation of quiescent cells with a serum pulse did not lead to an increased pERK1/2 and pAkt activity, suggesting a constitutive activation of Akt and ERK1/2 in thyroid cancer cells to an almost maximal extent.

Pharmacological inhibition of kinases. We first optimized the conditions to achieve maximal inhibition rates after drug application. Drugs were applied for $72 \mathrm{~h}$ either without further medium change, with one medium change after $36 \mathrm{~h}$ or with two medium changes after 24 and 48 h (Fig. 2). The most efficient inactivation of ERK1/2 kinases was achieved when U0126 or PD0325901 were added daily or at least once after $36 \mathrm{~h}$ (shown for U0126 in Fig. 2A). An almost complete inactivation of Akt over the whole three-day incubation period was obtained with a single addition of $1 \mu \mathrm{M}$ MK-2206 in B-CPAP and with a twice repeated addition in FTC-133 cells. In contrast, in Cal-62 only an incomplete inhibition was detected, even when Mk-2206 was renewed daily (Fig. 2B) or its concentration was increased to $2 \mu \mathrm{M}$ (not shown). Treatment with multikinase inhibitor sorafenib did not affect the activation of Akt and ERK1/2 in Cal-62 and FTC-133 cells, but strongly reduced the pERK1/2 expression level in B-CPAP cells at a concentration of $5 \mu \mathrm{M}$ (Fig. 2C). PI3K inhibitor wortmannin efficiently inhibited pAkt expression, but due to the instability of the drug, this effect was stable only for short time periods after application (Fig. 2D, compare incubation times of 4 and $24 \mathrm{~h})$. Such types of experiments also allow to analyze the presence of crosstalk between PI3K/Akt and the Ras/MAPK pathways. Indications for crosstalks of the two pathways were mostly observed after a more frequent drug application such as the upregulation of pERK1/2 upon MK-2206 application in B-CPAP cells or the downregulation of pERK1/2 upon 


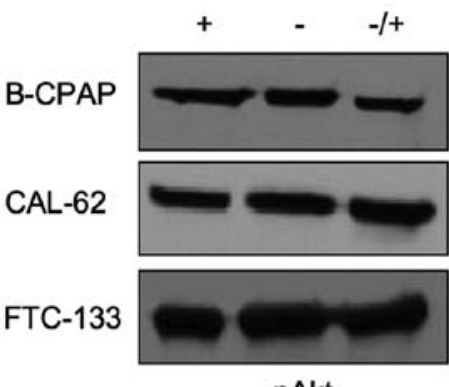

pAkt

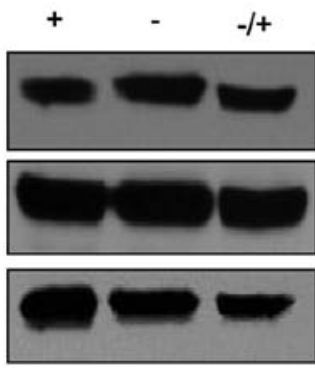

Akt

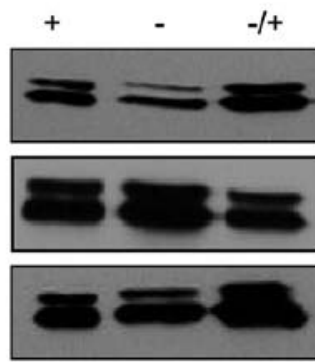

pERK1/2

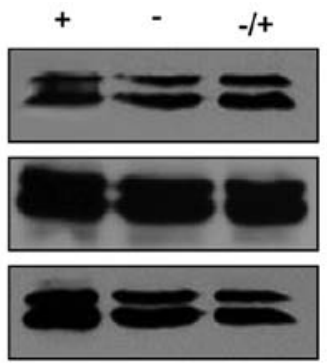

ERK1/2

Figure 1. Western blot analysis of Akt and ERK1/2 activation in thyroid carcinoma cells. Thyroid carcinoma cells B-CPAP, Cal-62 and FTC-133 were cultured for $48 \mathrm{~h}$ in DMEM, 10\% FCS (+), DMEM (-) or DMEM + 10\% FCS (-/+, only during the final 20 min of culture). Cell lysates were analyzed for the presence of total or phosphorylated (p) Akt or ERK1/2 proteins. Equal amounts of protein $(50 \mu \mathrm{g})$ were loaded per lane.
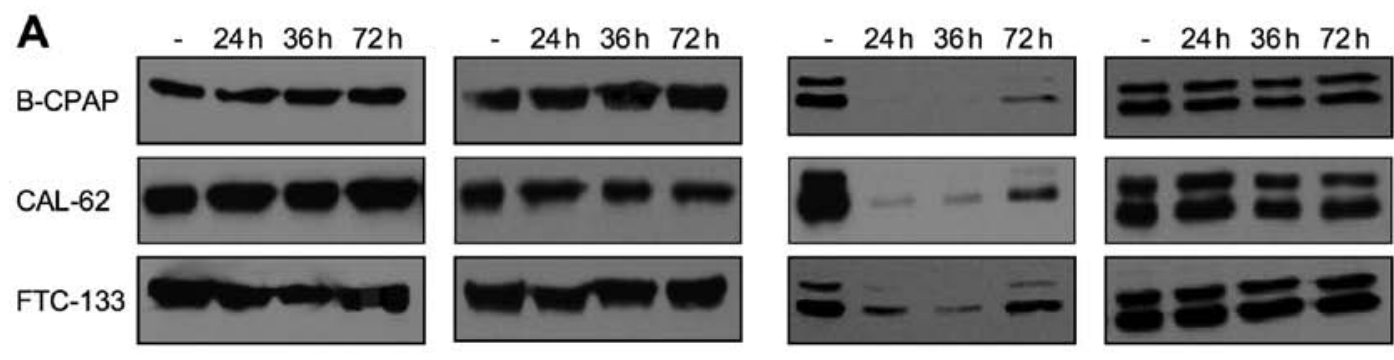

B

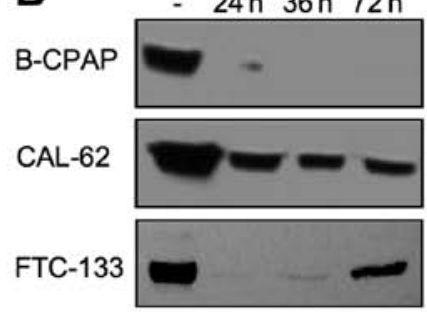

C

B-CPAP

CAL-62

FTC-133
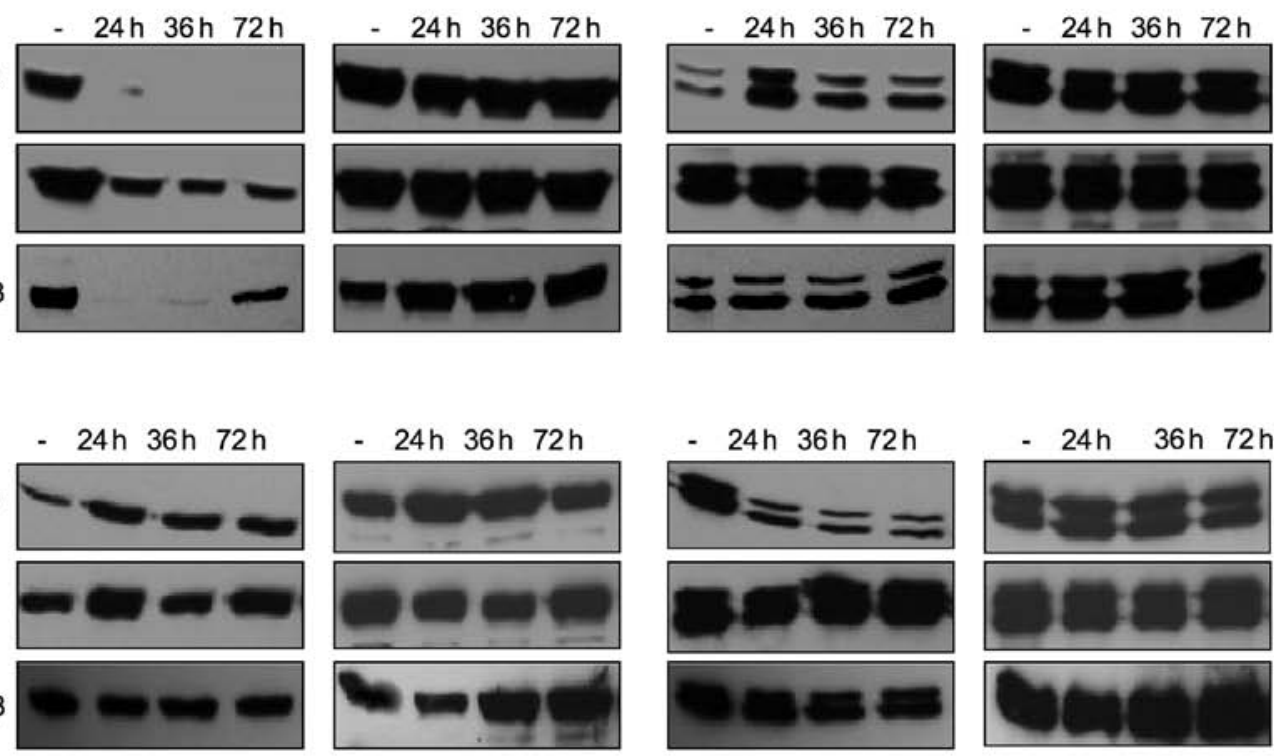

D
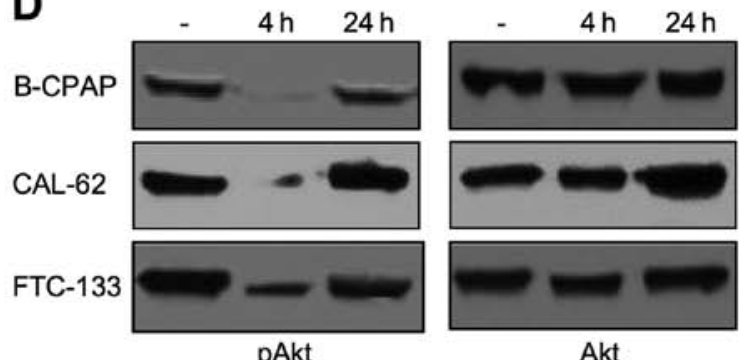

Akt

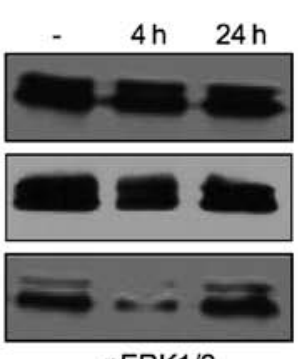

pERK1/2

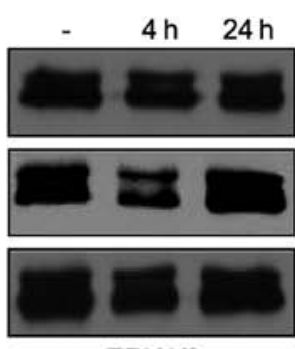

ERK1/2

Figure 2. Western blot analysis of pharmacological inhibition of Akt and ERK1/2 activation in thyroid carcinoma cells. Cells were treated with $20 \mu \mathrm{M}$ U0126 (A), $1 \mu \mathrm{M}$ MK-2206 (B) $5 \mu \mathrm{M}$ sorafenib (C) or $100 \mathrm{nM}$ wortmannin (D) under the following conditions. (A-C) Cells were cultured for $72 \mathrm{~h}$ without (-) or with additives, whereby the culture medium was exchanged every day $(24 \mathrm{~h})$, after half of the culture period $(36 \mathrm{~h})$ or remained unchanged (72 h).

(D) Cells were cultured $24 \mathrm{~h}$ in the absence (-) or for 4 or $24 \mathrm{~h}$ in the presence of $100 \mathrm{nM}$ wortmannin. Cell lysates where then analyzed for the activation, i.e., phosphorylation (p) level of Akt or ERK1/2 proteins. Equal amounts of protein (50 $\mu \mathrm{g})$ were loaded per lane. 


\section{B-CPAP}
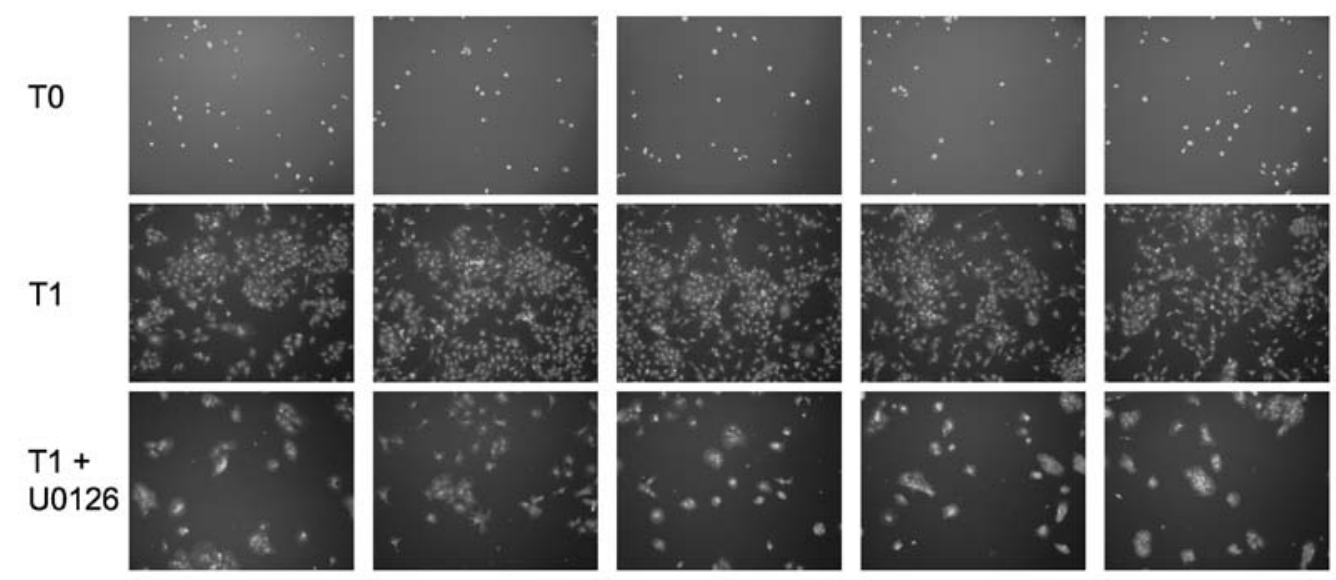

FTC-133
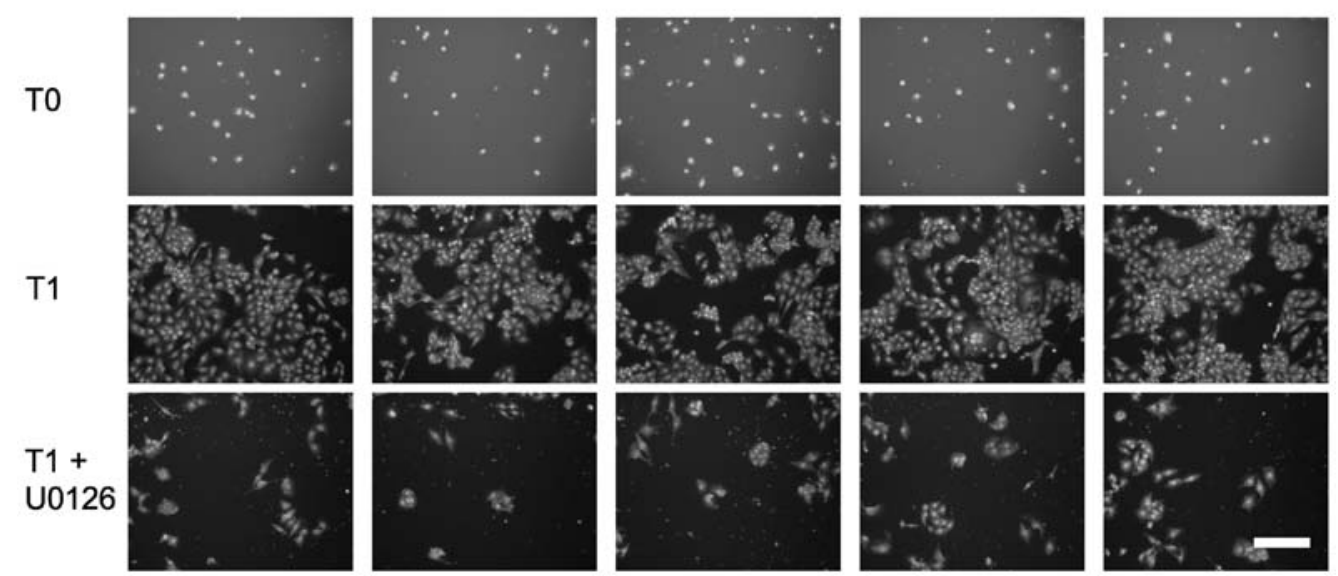

Figure 3. Impact of MEK/ERK1/2 inhibition on proliferation of thyroid carcinoma cells. Low-density B-CPAP or FTC-133 cells were cultured in 96-well plates for 0 (T0) or $72 \mathrm{~h}$ (T1) in the absence or presence of $20 \mu \mathrm{M}$ U0126, fixed with $4 \%$ formaldehyde and stained with DAPI. Representative micrographs of comparable areas in 5 different wells are shown for each time-point and treatment. Bar, $200 \mu \mathrm{m}$.

wortmannin treatment in FTC-133 cells (Fig. 2). Based on these results, in three-day lasting functional assays, drugs were replaced every $24 \mathrm{~h}$ and applied at a concentration of $1 \mu \mathrm{M}$ for PD0325901, MK-2206 and sorafenib and at $20 \mu \mathrm{M}$ for U0126. Because of its low stability, wortmannin was not included in long-lasting experiments.

Impact of kinase inhibition on cell proliferation. Low density thyroid carcinoma cells were allowed to proliferate in 96 -well plates for three days in the absence or presence of compounds. Different sets of cells were stained with DAPI before and after the 72-h incubation period and cell numbers were determined microscopically (Fig. 3 and Table I). Perturbation of the Ras/ MAPK pathway led to a strong reduction in cell number, whereby inhibition rates of $70-90 \%$ were observed for the different cell lines after U0126 treatment (Fig. 3 and Table I). For PD0325901, inhibition rates varied between 53\% for FTC-113 cells and $84 \%$ for B-CPAP cells (Table I). Also with the multikinase inhibitor sorafenib proliferation of thyroid carcinoma cells could be efficiently inhibited in the range of 70 to $~ 90 \%$ (Table I). Disruption of the PI3K/Akt pathway with MK-2206 inhibited the proliferation rate by $\sim 65 \%$ in B-CPAP and by $~ 40 \%$ in Cal-62 and FTC-133 cells.
Table I. Impact of kinase inhibition on the proliferation rate of thyroid carcinoma cells.

\begin{tabular}{llccc}
\hline Cell line & U0126 & PD0325901 & Sorafenib & MK-2206 \\
\hline B-CPAP & $90 \pm 8^{\mathrm{c}}$ & $84 \pm 2^{\mathrm{c}}$ & $91 \pm 17^{\mathrm{c}}$ & $65 \pm 24^{\mathrm{b}}$ \\
Cal-62 & $81 \pm 11^{\mathrm{c}}$ & $74 \pm 27^{\mathrm{b}}$ & $68 \pm 6^{\mathrm{c}}$ & $42 \pm 14^{\mathrm{b}}$ \\
FTC-133 & $72 \pm 8^{\mathrm{c}}$ & $53 \pm 13^{\mathrm{b}}$ & $69 \pm 6^{\mathrm{c}}$ & $43 \pm 16^{\mathrm{b}}$ \\
\hline
\end{tabular}

Cells were seeded at low density in 96-well plates and cultured for $72 \mathrm{~h}$ in the absence of drugs or in the presence of $20 \mu \mathrm{M} \mathrm{U} 0126,1 \mu \mathrm{M}$ PD0325901, $5 \mu \mathrm{M}$ sorafenib, $1 \mu \mathrm{M}$ MK-2206. For counting, fixed cells were stained with DAPI at the beginning and end of the culture period and the number of cells present in a defined area determined microscopically for 10 wells per time-point and treatment. Relative inhibition rate (in comparison to untreated cells in \%) was calculated from three independent experiments and mean values \pm SD are shown. Statistical significance was evaluated with an unpaired two-sample Student's t-test. ${ }^{\mathrm{a}} \mathrm{p}<0.05 ;{ }^{\mathrm{b}} \mathrm{p}<0.01 ;{ }^{\mathrm{c}} \mathrm{p}<0.001$.

Impact of kinase inhibition on collective cell migration. Thyroid carcinoma cells were seeded at high density on defined areas into Petri dishes and allowed to adhere for two 
A
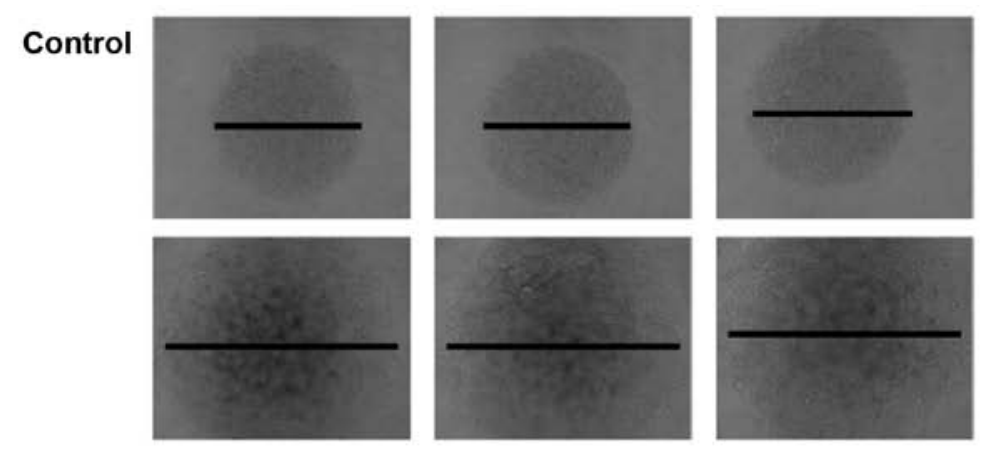

MK-2206
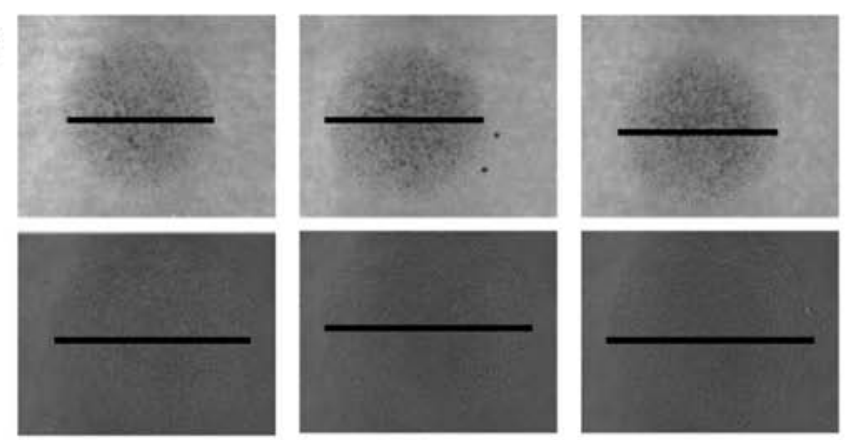

B

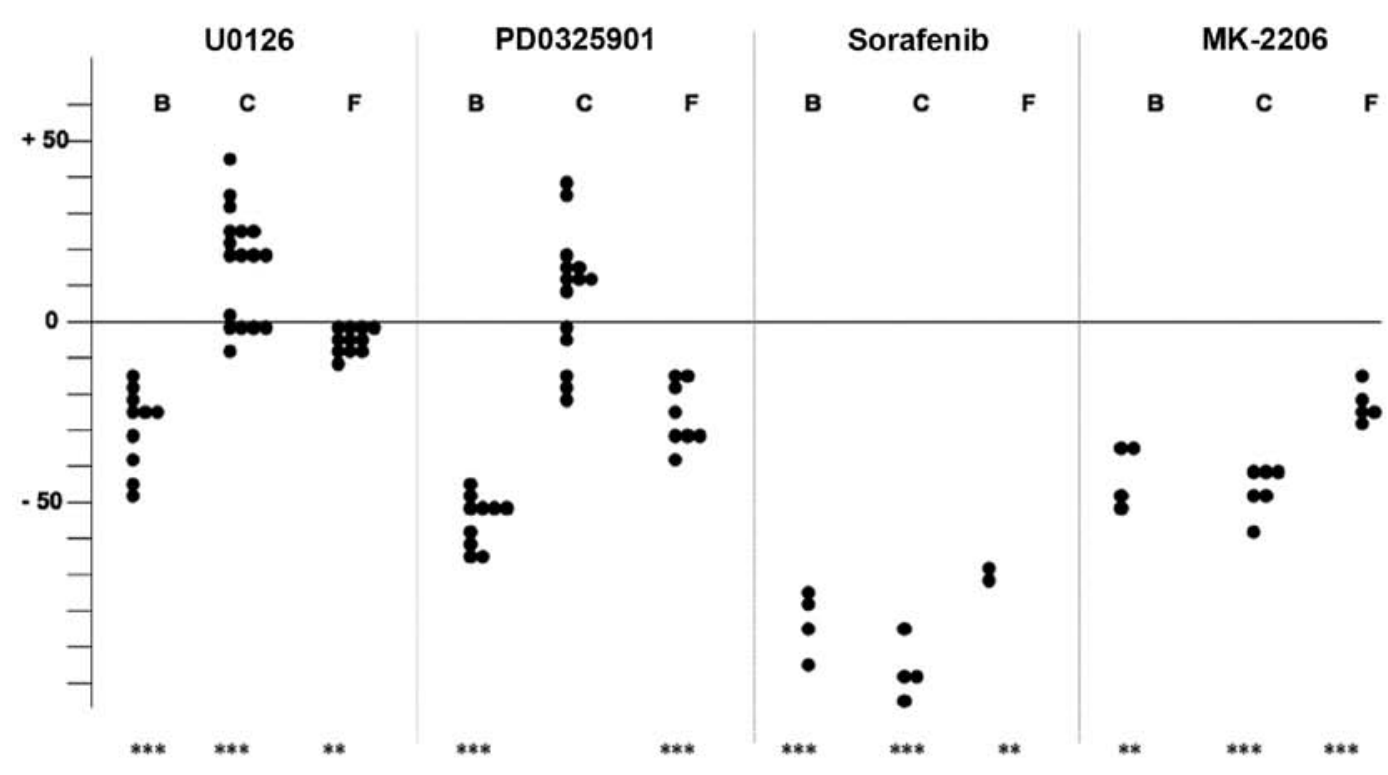

Figure 4. Impact of kinase inhibition on collective cell migration of thyroid carcinoma cells. Cells were forced to adhere as circular confluent monolayers (diameter 1-2 mm) on a plastic surface and allowed to migrate for $72 \mathrm{~h}$ in the absence or presence of additives. Diameters of monolayers were determined before (T0) and after (T1) the 72-h culture period. (A) Representative micrographs of three out of twelve untreated (control) or treated (MK-2206) monolayers of FTC-133 cells are shown at the beginning (T0) and end (T1) of the experiment. Bars, which document the maximal diameter of the circles are given for better orientation. (B) The graph documents the individual activation or inhibition rates of all experiments performed with B-CPAP (B), Cal-62 (C) and FTC-133 (F) cells. Each dot represents the average value calculated in one experiment for one individual cell line (given on the y-axis in percent as compared to control experiments without additives); ${ }^{* *} \mathrm{p}<0.01 ;{ }^{* * *} \mathrm{p}<0.001$.

to three hours to establish a circular monolayer. After floating with medium, cells were cultured for three days in the presence or absence of drugs (Fig. 4A for FTC-133 cells) and the diameters of the monolayers were determined at the beginning and the end of the culture period (Table II). In general, the migration capacity of thyroid carcinoma cells was suppressed to a varying extent in the presence of the different kinase inhibitors. For B-CPAP cells, inhibition rates in the range of 30 (for U0126) up to 80\% (for sorafenib) were observed. The migration capacity of FTC- 133 cells was relatively robust with regard to drug treatment (inhibition rates in the range of 7-50\%). Surprisingly, MEK/ERK1/2-inhibitor treated Cal-62 cells showed an increased migration rate, notwithstanding a strong inhibitory effect in the range of 45 or $80 \%$ became 

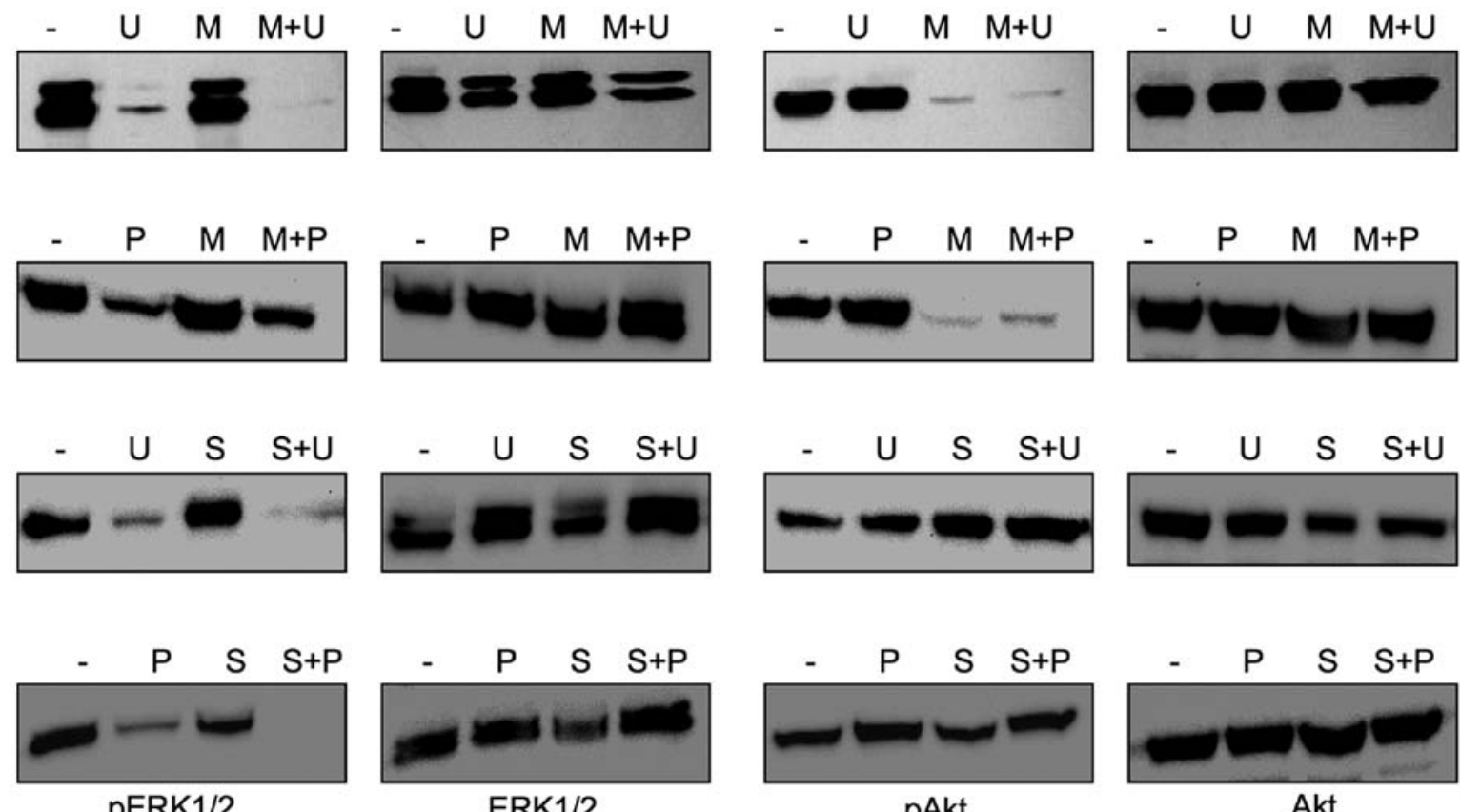

Figure 5. Western blot analysis of Akt and ERK1/2 activation in double-treated Cal-62 carcinoma cells. Cells were treated for $24 \mathrm{~h}$ with $1 \mu \mathrm{M}$ MK-2206 (M), $1 \mu \mathrm{M}$ PD0325901 (P), $5 \mu \mathrm{M}$ sorafenib (S) or $20 \mu \mathrm{M} \mathrm{U} 0126$ (U) as single additives or at the indicated combinations. Cell lysates were then analyzed for the activation, i.e., phosphorylation (p) level of Akt or ERK1/2 proteins. Equal amounts of protein $(50 \mu \mathrm{g})$ were loaded per lane.

Table II. Impact of drug treatment on collective cell migration of thyroid carcinoma cells.

\begin{tabular}{lcrcc}
\hline Cell line & U0126 & PD0325901 & Sorafenib & MK-2206 \\
\hline B-CPAP & $29 \pm 9^{\mathrm{c}}$ & $53 \pm 7^{\mathrm{c}}$ & $79 \pm 12^{\mathrm{c}}$ & $39 \pm 8^{\mathrm{b}}$ \\
Cal-62 & $+15 \pm 12^{\mathrm{c}}$ & $+10 \pm 17$ & $84 \pm 12^{\mathrm{c}}$ & $46 \pm 7^{\mathrm{c}}$ \\
FTC-133 & $7 \pm 5^{\mathrm{b}}$ & $23 \pm 9^{\mathrm{c}}$ & $53 \pm 3^{\mathrm{b}}$ & $24 \pm 6^{\mathrm{c}}$ \\
\hline
\end{tabular}

Cells at high density $(15,000$ cells in $3 \mu 1)$ were seeded onto twelve defined regions per Petri dish and allowed to adhere for two to three hours. Dishes were then floated with culture medium (T0) and cells were treated for three days (T1) with or without additives. Diameters of circular areas occupied by cells (Fig. 4) were determined at T0 and T1. Two to seventeen experiments were performed for each cell line. Values (given as mean $\pm \mathrm{SD}$ ) represent inhibition rates in percent (in respect to circle diameter of controls). Statistical significance was evaluated with an unpaired two-sample Student's t-test. ${ }^{\mathrm{a}} \mathrm{p}<0.05$; ${ }^{\mathrm{b}} \mathrm{p}<0.01 ;{ }^{\mathrm{c}} \mathrm{p}<0.001$.

visible upon MK-2206 or sorafenib treatment. Eventhough statistical significance is only present for U0126-treatment, the data shown in Fig. 4B demonstrate that in more than half of the experiments performed with PD0325901 an increased migration also took place.

We thus wondered, if the stimulating effect of U0126 and PD0325901 on the migration of Cal-62 cells could be suppressed by a simultaneous inhibition of the PI3K/Akt pathway, because western blot analyses revealed that the combined treatment of thyroid carcinoma cells with U0126 and MK-2206 led to an almost complete downregulation of pAkt and pERK1/2 expression (Fig. 5). Indeed, the migration rate of U0126/MK-2206 dual-treated Cal-62 cells was substantially inhibited not only
Table III. Impact of single or dual drug treatment on collective cell migration of Cal-62 cells.

\begin{tabular}{lccc}
\hline A. & & & \\
\hline Additive & - & U0126 & PD0325901 \\
\hline- & 0 & $+23 \pm 16^{\mathrm{b}}$ & $+4 \pm 18$ \\
MK-2206 & $46 \pm 7^{\mathrm{c}}$ & $34 \pm 6^{\mathrm{c}}$ & $52 \pm 8^{\mathrm{c}}$ \\
Sorafenib & $84 \pm 12^{\mathrm{c}}$ & $54 \pm 7^{\mathrm{c}}$ & $33 \pm 21^{\mathrm{b}}$ \\
\hline
\end{tabular}

B.

\begin{tabular}{|c|c|c|c|}
\hline Additive & - & U0126 & PD0325901 \\
\hline - & 0 & $+36 \pm 6^{\mathrm{b}}$ & $+18 \pm 5^{\mathrm{b}}$ \\
\hline $\begin{array}{l}\text { Sorafenib } \\
2 \mu \mathrm{M}\end{array}$ & $14 \pm 4^{b}$ & $2 \pm 7$ & $12 \pm 9$ \\
\hline $\begin{array}{l}\text { Sorafenib } \\
1 \mu \mathrm{M}\end{array}$ & $7 \pm 9$ & $1 \pm 5$ & $8 \pm 2$ \\
\hline
\end{tabular}

Cal-62 cells were seeded and treated as described in legend of Fig. 4. Values are given as mean \pm SD of up to five (A) or three (B) independent experiments and represent inhibition rates in percent (in respect to diameters of circles in control experiments). Values for single or double treatments are shown. Statistical significance in respect to control treatment without additives was evaluated with an unpaired two-sample Student's t-test: ${ }^{\mathrm{a}} \mathrm{p}<0.05 ;{ }^{\mathrm{b}} \mathrm{p}<0.01 ;{ }^{\mathrm{c}} \mathrm{p}<0.001$.

in respect to U0126- or PD0325901-single-treated, but also in comparison to untreated control cells (Table IIIA). Moreover, although sorafenib did not affect Akt activation in U0126- or 

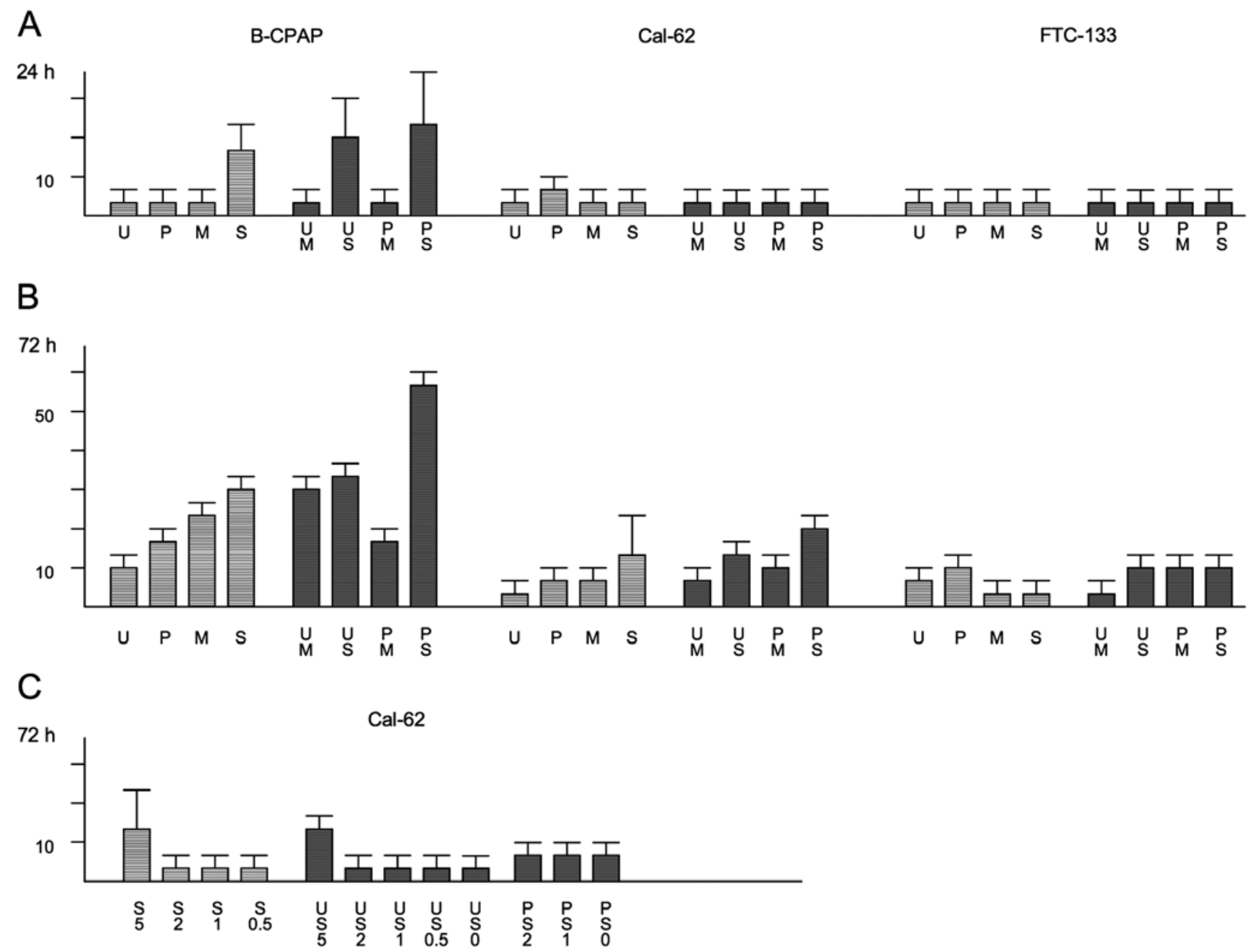

Figure 6. Cytotoxic effects of kinase inhibitors on thyroid carcinoma cells as determined by LDH assay. Cells were cultivated for $24 \mathrm{~h}$ (A) or $72 \mathrm{~h}$ (B and C) either in the absence of additives or in the presence of $1 \mu \mathrm{M} \mathrm{MK}-2206$ (M), $1 \mu \mathrm{M}$ PD0325901 (P), $5 \mu \mathrm{M}$ sorafenib (S) or $20 \mu \mathrm{M}$ U0126 (U) as single additives or at the indicated combinations and concentrations [given in $\mu \mathrm{M}$ for sorafenib (C)]. Relative LDH activity present in the cell culture supernatant (rate of cytotoxicity) are shown. Data are means \pm SD from two to three independent experiments.

PD0325901-treated Cal-62 cells (Fig. 5), its presence led to a substantial inhibition of cell migration (Table IIIA). To rule out that the inhibition observed results mainly from cytotoxic drug effects, LDH assays were carried out. After a 24-h incubation period none of the single or dual drug treatments induced a noteworthy cytotoxic effect in Cal-62 cells, although dual-treated B-CPAP cells were considerably affected (Fig. 6A). After a 72-h incubation period, however, a moderate cytotoxic effect was observed in sorafenib-treated Cal-62 cells. Therefore, it is possible that under these conditions the proliferation and migration suppressing potential of this compound is partially due to a cytotoxic effect. Thus, in a final set of experiments, we applied sorafenib at concentrations of 1 and $2 \mu \mathrm{M}$, i.e., at concentrations that did not induce considerable cytotoxic effects in Cal-62 cells after a 72-h incubation period neither as a single agent nor in combination with $20 \mu \mathrm{M}$ U0126 or $2 \mu \mathrm{M}$ PD0325901 (Fig. 6C). Under these conditions, the migration-promoting effect of U0126 was completely compensated, but an additional inhibition, as could be monitored for higher sorafenib concentrations, was not observed (Table IIIB). Altogether, these data suggest that the migration-stimulating effect of PD0325901 and U0126 can be suppressed by Akt-dependent and -independent mechanisms, even though an inactivation of Akt downstream signaling molecules cannot be completely excluded.

\section{Discussion}

Our study demonstrated that the migration and proliferation of human thyroid carcinoma cells is partially mediated via constitutive active PI3K/Akt and RAS/MAPK signaling. Pharmacological inhibition of this signaling considerably inhibits proliferation in all three cell lines, although to varying extent (Table I). In general, also the migration rate of thyroid carcinoma cells is suppressed in the presence of the different kinase inhibitors, with the remarkable exception of an increased migration of Cal-62 cells in the presence of U0126- or PD0325901. This increase can be prohibited by MK-2206 treatment, i.e., via Akt inactivation, or by application of sorafenib, a compound that does not perturb Akt activation level in Cal-62 cells.

Crosstalk of signaling pathways in thyroid carcinoma cells. PI3K/Akt and Ras/MAPK signaling pathways, although 
first described as linear conduits were soon shown to be connected with each other in a complicated fashion that comprises cross-activation and -inhibition processes as well as negative feedback loops and pathway convergence (18). In our experiments we found evidence for the presence of crossinhibitory circuits of PI3K/Akt on Ras/MAPK in B-CPAP and of cross-activating circuits in FTC-133 cells (Fig. 2). As such crosstalk depends inter alia on substrate availability that can vary between different cell types or cell lines it is not surprising that the interactions we observed exhibit a cell linespecific pattern that can not be generalized (Fig. 2). Kandil and colleagues (19) also recently reported that cross-activation of Akt varies between different thyroid cancer cell lines upon treatment with the MEK/ERK inhibitor AZD6244. At the present level of knowledge, it is mainly assumed that the PI3K/ Akt and Ras/MAPK pathways can negatively regulate each other and, in addition, the latter one can activate the former (18). Nevertheless, and thereby underlining our data, in a few reports it has been shown, that the PI3K/Akt pathway can activate ERK1/2 signaling (20-22).

Yin-yang effects in thyroid carcinoma cells. Although we have evidence for the presence of crosstalk between PI3K/Akt- and Ras/MAPK pathways in thyroid carcinoma cells, it does not illuminate the yin-yang effect we detected in Cal-62 cells treated with MEK inhibitors:

i) Treatment of Cal-62 cells with MEK inhibitors leads to an almost complete inhibition of pERK1/2 expression, but leaves pAkt expression substantially unaffected (Fig. 2). Vice versa, treatment with an Akt inhibitor strongly decreases pAkt expression without affecting the expression level of pERK1/2 (Fig. 2). Thus, no obvious crosstalk of the two pathways seems to be present in Cal-62 cells.

ii) The proliferation rate of Cal-62 cells is strongly suppressed by MEK inhibitors, but only mildly by MK-2206 (Table I), whereas the migration rate is significantly enhanced by MEK inhibitors and strongly suppressed by MK-2206 (Table II). Thus, the increased surface area observed in the collective cell migration experiments cannot be simply the result of an increased cell mass, or, more generally, of a growth process.

iii) Increased migration of Cal-62 cells due to the presence of MEK inhibitors can be suppressed by MK-2206 or sorafenib, i.e., in the presence of low as well as high expression levels of pAkt. To our knowledge, sorafenib treatment does not affect Akt downstream signaling molecules. Thus, it is likely that the stimulatory effect of MEK inhibitors can be suppressed via Akt-dependent and -independent signaling mechanisms.

Collectively, it seems feasible that interference with a single pathway can inhibit one cellular process, here the cell's proliferative capacity, and at the same time promote another, here the cell's migratory potential. In our example such an effect can be interpreted by assuming the existence of an (hyper)activated Ras/ERK1/2 pathway that suppresses cell migration but can itself be suppressed by MEK inhibitors. Based on the above, one could expect that Cal-62 cells migrate with a submaximal velocity, and indeed, although we have not performed a systematic analysis, Cal-62 shows the lowest velocity amongst the three cell lines we have used, i.e., a 25 or $40 \%$ reduced migration rate in comparison to FTC-133 or B-CPAP cells, respectively. This resembles a scenario that hyperactivation of the Ras/ERK1/2 pathway can trigger a decreased proliferation rate, i.e., cell cycle arrest (23). An inhibitory effect of the MAPK cascade on cell proliferation is also active in K562 erythroleukemia cells, which differentiate into megakaryocytes upon phorbol ester stimulation, a process that is: i) accompanied by growth retardation and ERK activation, and ii) completely suppressed in the presence of MEK inhibitors (24). The aspect that Ras/ ERK1/2 pathway activation not only activates $(25,26)$, but may also suppress cell migration adds another facet to the complex pattern of MAPK signaling. To overcome the stimulatory effect of MEK inhibitors on Cal-62 cells, we have performed dual treatments to interfere with more than one signaling pathway and, thereby, successfully suppressed cell proliferation as well as migration. Such a strategy, in clinical practice known as a subtype of combination therapy (27), has been used in a number of trials to attack the fatal potential of tumor cells more efficiently. For example, in thyroid carcinoma cells, dual Ras/MAPK and PI3K/Akt/mTOR pathway inhibition leads to significant higher growth retardation even in a synergistic manner when compared to single pathway inhibition $(28,29)$. Beside the advantage of a combinatorial treatment to increase the inhibitory action of a single drug such a strategy also decreases the risk of unfavorable yin-yang effects.

\section{Acknowledgements}

J.W. was supported by a grant from the German Research Foundation and the Medical Faculty of the University of Bonn (CRU208/TP10).

\section{References}

1. Ambrosetti MC, Colato C, Dardano A, Monzani F and Ferdeghini M: Radioiodine ablation: when and how. Q J Nucl Med Mol Imaging 53: 473-481, 2009.

2. Hannallah J, Rose J and Guerrero MA: Comprehensive literature review: recent advances in diagnosing and managing patients with poorly differentiated thyroid carcinoma. Int J Endocrinol 2013: 317487, 2013.

3. Dar AC and Shokat KM: The evolution of protein kinase inhibitors from antagonists to agonists of cellular signaling. Annu Rev Biochem 80: 769-795, 2011

4. Gild ML, Bullock M, Robinson BG and Clifton-Bligh R: Multikinase inhibitors: a new option for the treatment of thyroid cancer. Nat Rev Endocrinol 7: 617-624, 2011.

5. Antonelli A, Fallahi P, Ferrari SM, et al: Dedifferentiated thyroid cancer: a therapeutic challenge. Biomed Pharmacother 62: 559-563, 2008 .

6. Nikiforov YE: Thyroid carcinoma: molecular pathways and therapeutic targets. Mod Pathol 21: S37-S43, 2008

7. Saji $M$ and Ringel MD: The PI3K-Akt-mTOR pathway in initiation and progression of thyroid tumors. Mol Cell Endocrinol 32: $20-28,2010$.

8. Wilhelm SM, Adnane L, Newell P, Villanueva A, Llovet JM and Lynch M: Preclinical overview of sorafenib, a multikinase inhibitor that targets both Raf and VEGF and PDGF receptor tyrosine kinase signaling. Mol Cancer Ther 7: 3129-3140, 2008.

9. Duntas LH and Bernardini R: Sorafenib: rays of hope in thyroid cancer. Thyroid 2: 1351-1358, 2010.

10. Fallahi P, Ferrari SM, Santini F, et al: Sorafenib and thyroid cancer. BioDrugs 27: 615-628, 2013.

11. Chai H, Luo AZ, Weerasinghe P and Brown RE: Sorafenib downregulates ERK/Akt and STAT3 survival pathways and induces apoptosis in a human neuroblastoma cell line. Int J Clin Exp Pathol 3: 408-415, 2010.

12. Carlo-Stella C, Locatelli SL, Giacomini A, et al: Sorafenib inhibits lymphoma xenografts by targeting MAPK/ERK and AKT pathways in tumor and vascular cells. PLoS One 8: e61603, 2013. 
13. Nguyen TK, Jordan N, Friedberg J, Fisher RI, Dent P and Grant S: Inhibition of MEK/ERK1/2 sensitizes lymphoma cells to sorafenib-induced apoptosis. Leuk Res 34: 379-386, 2010.

14. Rose A, Grandoch M, vom Dorp F, Rübben H, Rosenkranz A, Fischer JW and Weber AA: Stimulatory effects of the multikinase inhibitor sorafenib on human bladder cancer cells. Br J Pharmacol 160: 1690-1698, 2010.

15. Gedaly R, Angulo P, Hundley J, Daily MF, Chen C and Evers BM: PKI-587 and sorafenib targeting PI3K/AKT/mTOR and Ras/Raf/ MAPK pathways synergistically inhibit HCC cell proliferation. J Surg Res 176: 542-548, 2012.

16. Fremin $C$ and Meloche S: From basic research to clinical development of MEK1/2 inhibitors for cancer therapy. J Hematol Oncol 3: Feb 11, 2010. doi:10.1186/1756-8722-3-8.

17. Glassmann A, Reichmann K, Scheffler B, Glas M, Veit N and Probstmeier R: Pharmacological targeting of the constitutively activated MEK/MAPK-dependent signaling pathway in glioma cells inhibits cell proliferation and migration. Int J Oncol 39: 1567-1575, 2011.

18. Mendoza MC, Er EE and Blenis J: The Ras-ERK and PI3K-mTOR pathways: cross-talk and compensation. Trends Biochem Sci 36: 320-328, 2011.

19. Kandil E, Tsumagari K, Ma J, et al: Synergistic inhibition of thyroid cancer by suppressing MAPK/PI3K/AKT pathways. J Surg Res 184: 898-906, 2013.

20. Hong SK, Jeong JH, Chan AM and Park JI: AKT upregulates B-Raf Ser445 phosphorylation and ERK1/2 activation in prostate cancer cells in response to androgen depletion. Exp Cell Res 319: $1732-1743,2013$
21. Niba ET, Nagaya H,Kanno T, et al: Crosstalk between PI3 kinase/ PDK1/Akt/Racl and Ras/Raf/MEK/ERK pathways downstream PDGF receptor. Cell Physiol Biochem 31: 905-913, 2013.

22. Wang CC, Cirit M and Haugh JM: PI3K-dependent cross-talk interactions converge with Ras as quantifiable inputs integrated by Erk. Mol Syst Biol 5: 246, 2009.

23. Meloche $S$ and Pouysségur J: The ERK1/2 mitogen-activated protein kinase pathway as a master regulator of the G1- to S-phase transition. Oncogene 26: 3227-3239, 2007.

24. Whalen AM, Galasinski SC, Shapiro PS, Nahreini TS and Ahn NG: Megakaryocytic differentiation induced by constitutive activation of mitogen-activated protein kinase kinase. Mol Cell Biol 17: 1947-1958, 1997.

25. Chen H, Zhu G, Li Y, et al: Extracellular signal-regulated kinase signaling pathway regulates breast cancer cell migration by maintaining slug expression. Cancer Res 69: 9228-9235, 2009.

26. Huang $C$, Jacobson $K$ and Schaller MD: MAP kinases and cell migration. J Cell Sci 117: 4619-4628, 2004

27. Knight ZA, Lin H and Shokat KM: Targeting the cancer kinome through polypharmacology. Nat Rev Cancer 10: 130-137, 2010.

28. Jin N, Jiang T, Rosen DM, Nelkin BD and Ball DW: Dual inhibition of mitogen-activated protein kinase kinase and mammalian target of rapamycin in differentiated and anaplastic thyroid cancer. J Clin Endocrinol Metab 94: 4107-4112, 2009

29. Liu D and Xing M: Potent inhibition of thyroid cancer cells by the MEK inhibitor PD0325901 and its potentiation by suppression of the PI3K and NF-kappaB pathways. Thyroid 18: 853-864, 2008. 